\title{
Smooth Switching between 2-stroke and 4-stroke Modes of HCCI Operation
}

\author{
Maxim V. Subbotin, Sungbae Park, Aleksandar Kojic, Jasim Ahmed, Nalin Chaturvedi, David Cook
}

\begin{abstract}
Switching between 2-stroke and 4-stroke modes of Homogeneous Charge Compression Ignition (HCCI) operation is a promising method for extending load range of HCCI engine. Switching between the two modes introduces disturbances into the system resulting in significant tracking errors. We propose an architecture for control system and a method for calculation of control inputs which minimize the tracking errors during switching. Proposed method produces inputs for engine valve timings which insure smooth transitions between the two HCCI modes under a varying engine load. The method uses techniques from the theory of system identification and the theory of optimal control.
\end{abstract}

\section{INTRODUCTION}

Homogeneous Charge Compression Ignition (HCCI) technology shows an improvement in efficiency and significant reduction in emissions in comparison to Spark Ignition (SI) and Compression Ignition (CI) internal combustion technologies. The improved fuel economy of the HCCI engines is explained by a high dilution of charge and low throttling losses which simultaneously results in lower Carbon Monoxide $(C O)$ emissions [3], [5], [8]. Reduction of Nitrous Oxides $\left(N O_{x}\right)$ emissions by about two orders of magnitude in comparison to conventional SI and CI diesel engines is explained by much lower combustion temperatures due to the homogeneous nature of the combustion process [5], [7].

One of the main challenges in achieving the HCCI regime is an initiation of the homogeneous combustion. The HCCI engine unlike the SI or CI engines where combustion is initiated with spark or fuel injection lacks a specific event initiating combustion. The auto ignition of a premixed charge in the cylinder is achieved by controlling concentrations of the mixture components and their temperature at the beginning of a combustion stroke. Two main approaches for control of the mixture temperature are heating or precompressing of an intake charge [9], [16] and reinduction or trapping exhaust gasses from the previous cycle [1], [2], [13], [14]. The latter approach results in higher overall efficiency and is implemented using active valve control system regulating valve timing.

The high dilution of fresh charge with exhaust gasses in the engine cylinder is required to increase temperature at the beginning of the combustion stroke. The dilution results in a limited work output of the HCCI engine in comparison to

\footnotetext{
M. V. Subbotin, S. Park, A. Kojic, J. Ahmed N. Chaturvedi, and D. Cook, RTC-EMCC, Robert Bosch LLC, subbotindengineering.ucsb.edu (sungbae.park, aleksandar.kojic, jasim.ahmed, nalin. chaturvedi, david.cook) dus.bosch.com
}

SI and CI engines. Several methods are introduced to overcome this limitation. One of the methods employs a hybrid switching strategy which involves switching between HCCI and standard SI modes [10], [11]. When the load is low the engine can operate in a highly efficient HCCI mode. When a higher output is required the engine switches to the SI mode, though the advantages of the HCCI are lost. Another alternative allowing the engine to operate in the broad range of loads, is a hybrid strategy involving switching between 4-stroke and 2-stroke HCCI modes [6], [12]. This method allows to harvest the efficiency and emission advantages of the HCCI technology without limiting of the load range. In addition to that the switching between the two HCCI modes is potentially easier to implement than the switching between the 4-stroke HCCI and the SI modes. The main idea of the method is to use the 4-stroke HCCI mode at low loads and switch to the 2-stroke mode at high loads to double the number of combustion events per engine revolution while preserving all the advantages of the HCCI technology.

Control inputs used to track desired reference signals in the two modes are different due to the differences in the gas exchange processes for the 2-stoke and the 4-stroke modes [1], [4], [5], [14], [15]. It is natural to assume that for each of the two modes we design and tune a mode-specific controller which regulates operation of the engine. If engine operation is controlled by two controllers, one for each mode, tuned and optimized to provide desired engine performance at the specific mode, the controllers may not be able to eliminate the effects of disturbances introduced during switching. As a consequence, switching between the two modes may result in significant tracking errors in engine outputs if the influence of the disturbances on the engine outputs is not counted for.

In this paper we propose a modular architecture for the control system which allows the use of two mode-specific controllers, one for the 2-stroke mode and one for the 4stroke mode, and introduce an auxiliary module which adds correction signals to the control inputs during switching in order to compensate for the disturbances. The modular structure facilitates a separation between the tasks for the two control modules, the mode-specific controller ensures local tracking while the correction module eliminates the effects of the disturbance. We propose a method for calculation of the correction signals generated by the correction module. The inputs used to provide smooth transitions between the two modes are the engine valve timings. The engine outputs which have to track desired reference signals are an Integrated Mean Effective Pressure (IMEP) and a combustion phasing. Proposed method for calculation of the correction 
signals uses basic techniques from the theory of the system identification and the theory of optimal control.

We design the proposed control system and develop the method using a 19-th order nonlinear model of the HCCI engine [15] which can switch between the 2-stroke and the 4-stroke modes. We assume that the switching between the two modes is controlled by a logic which triggers the engine to switch from one mode to another depending on the desired engine output. In the following sections we describe architecture of the proposed control system and introduce the method for computation of the correction signals. At the end we validate the proposed method through a simulation.

\section{Problem Motivation}

To study behavior of an engine switching between the 2stroke and the 4-stroke HCCI modes we use a nonlinear model developed in [15]. The model contains 19 states describing the evolution of temperatures and concentrations of chemical species in three volumes - intake volume, cylinder, and exhaust volume. The model was validated by comparison with experimental results from the engine operating in the 2-stroke and the 4-stroke modes. In the following development we use the word engine to refer to the nonlinear model. We assume that in each of the two modes the engine is controlled by the mode-specific controller and introduce the correction module which helps the controllers during switching. For illustrative purposes we assumed that the engine was controlled in the 2-stroke mode by the controller presented in [15] and designed a similar PI controller with a feedforward mapping for the 4-stroke mode. We tuned the 4-stroke controller to ensure that it provides good reference tracking of the IMEP and the combustion phasing signals. Since the switching strategy presented here does not depend on a particular implementation of the modespecific controllers we do not discuss development of these controllers. To show performance of the developed method, we implemented control logic which forced the engine to switch to the 2-stroke mode when the desired output in IMEP was greater or equal to 4.2 bar, and to switch back to the 4-stroke mode when the desired output was less or equal to 3.8 bar. This switching logic essentially implemented a hysteresis relay necessary to avoid chattering between the two HCCI modes. The combustion phasing in both modes had to be regulated to 7 degrees TDC and was measured at the middle point of the combustion reaction (MFB50). It should be clear that due to the double frequency of the combustion events in the 2-stroke mode, the effective IMEP in this mode is double of the IMEP for one combustion stroke.

To observe behavior of the engine switching between the two modes, consider Figure 1. The figure shows ramp reference signal in IMEP and constant reference in MFB50 and the corresponding engine outputs during switching between the two modes. The mode-specific controllers tuned to provide tracking of reference signals in the particular mode are not able to compensate for the disturbances introduced during switching and without any additional correction switching between the 2-stroke and the 4-stroke modes results in significant oscillations in engine outputs. We will call switching between the modes without any correction a hard switching in comparison to smooth switching when changing the mode of engine operation does not have any influence on the engine outputs. The oscillations in outputs occur mainly due to differences between gas temperatures and concentrations of species inside engine cylinder corresponding to the same outputs for the 2 -stroke and the 4-stroke modes. To observe that consider Table 1 which compares final conditions for 19state HCCI engine model. The table shows the in-cylinder temperatures and the concentrations at the end of the engine cycle for the two modes with identical engine outputs. As can be seen from the table, the in-cylinder temperature and concentrations of the exhaust gases at the end of the 4stroke cycle are much higher than the corresponding states at the end of the 2-stroke cycle. At the switching from the 4stroke to the 2-stroke mode the first 2-stroke cycle following the last 4-stroke cycle is started with the initial conditions which differ from the initial conditions corresponding to the normal 2-stroke operation with the engine producing the same output. As a consequence switching from the 4stroke to the 2-stoke mode results in a drop in IMEP and combustion phasing. Similar analysis is applicable to the switching from the 2-stroke to the 4-stroke mode which results in a jump in the IMEP and the combustion phasing.
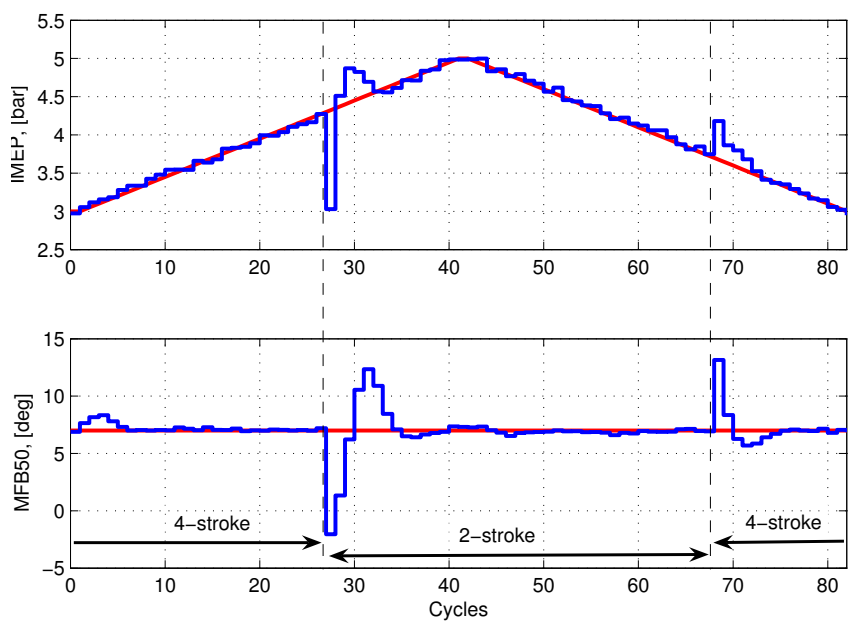

Fig. 1. HCCI engine tracking ramp reference signal in IMEP and constant reference (7 deg TDC) in MFB50 with switching between 2-stroke and 4stroke modes. Hard switching - no correction is implemented to insure a smooth transition between the modes.

Table 1: Temperatures $[\mathrm{K}]$ and concentrations of species $[\mathrm{mg} / \mathrm{kmol}]$

Table 1: Temperatures [K] and concentrations of species [mg/kmol]
\begin{tabular}{|c|ccccc|}
\hline & $T_{c}$ & $\mathrm{O}_{2}$ & $\mathrm{~N}_{2}$ & $\mathrm{CO}_{2}$ & $\mathrm{H}_{2} \mathrm{O}$ \\
\hline 2-stroke, 1.9 bar IMEP & 472.7 & 0.00265 & 0.01881 & 0.00151 & 0.00170 \\
\hline 4-stroke, 3.8 bar IMEP & 601.4 & 0.00265 & 0.02189 & 0.00203 & 0.00228 \\
\hline
\end{tabular}

\section{SOLUTION}

The differences between the temperatures and concentrations resulting in hard switching can be treated as disturbances acting on the system during the first engine cycle after switching and influencing the following cycles. In order 
to insure a smooth transition between the two modes we introduce a correction to system inputs, valve timings, which try to annihilate the influence of the disturbances on the system outputs. When switching takes place, we correct the inputs controlling the engine working at a specific mode with an additional signal. Correction takes place during several engine cycles after switching until the influence of the switching disturbance on the engine output is completely eliminated and the mode-specific controller is able to provide a desired output tracking. The resulting control system incorporating the mode-specific controller and a correcting controller active after the switching, is represented in Figure 2. While the mode-specific controller is closed in the feedback loop and its outputs depend on the engine output, the signals generated by the correcting controller depend only on the reference input. The correcting controller generates input sequence only during several cycles after switching and is inactive otherwise. Generation of the correction sequence is triggered by logic enforcing switching from one HCCI mode to another.

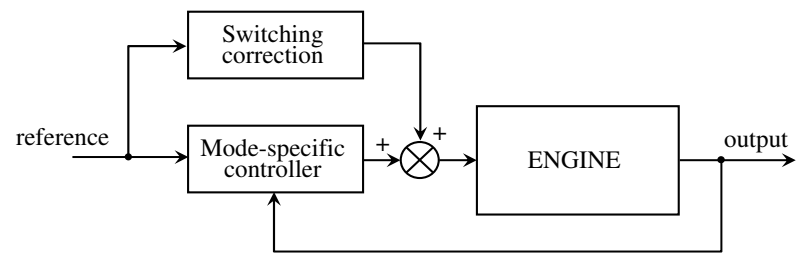

Fig. 2. Control system structure.

In order to find the correction sequences which minimize the influence of the switching disturbance on the engine outputs we develop a method which combines techniques from the theory of system identification and the theory of optimal control. As the first step in our development we find inputs which show a significant influence on the engine outputs in each mode after switching. Once the important engine inputs are defined, we identify two linear engine models, one model for each mode. The models are calculated by running a standard system identification technique applying excitation signals to the system, collecting the system response, and calculating the model based on the input-output data. For each of the two modes we calculate one linear time-invariant state-space model using a subspace identification methodology. While collecting the data necessary for identification, we apply the excitation inputs to the engine controlled in the 2-stroke or the 4-stroke mode by the corresponding mode-specific controller. Hence two linear models represent not models of the engine operating at certain points, but models of two closed-loop systems engine controlled by one of the controllers corresponding to the specific mode. Figure 3 shows a structure for identification of the two linear closed-loop system models.

Each identified system model defines a mapping between two vectors - a vector of initial conditions and a vector of correction signals applied to controlled engine - and a vector of modified engine outputs. If we know the initial conditions of the linear model corresponding to one of the modes, we can define a desired system output and formulate the optimization problem of minimizing the cost function weighting the influence of the switching disturbance on the engine output. We can find the minimum of the cost function by solving a least squares problem and find the optimal input - a correction sequence minimizing the influence of disturbance on the IMEP and the combustion phasing. The initial conditions of each linear model at the first cycle after switching are defined by the switching points between the two modes. These initial conditions essentially model a state of the engine at the beginning of the first new mode engine cycle. To find initial conditions corresponding to a particular switching point and one of the two linear models, we need to record the output of the engine during hard switching and solve a least squares problem with the uncorrected engine response.

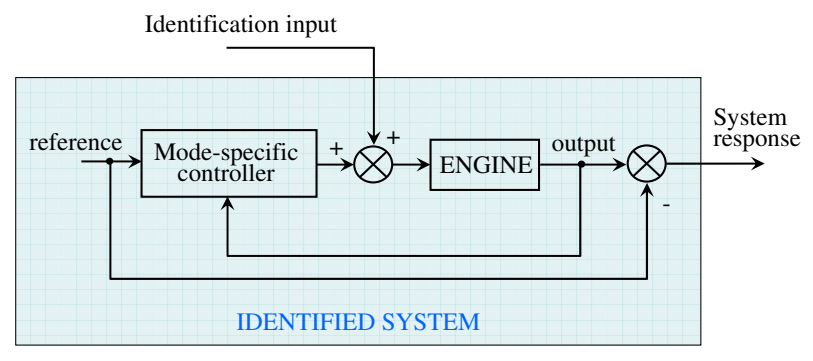

Fig. 3. Identification structure.

We start our development from defining the inputs which have strong influence on the system response. In order to find these inputs we first consider two boundary engine cycles, the last cycle before switching and the first cycle after switching. Figure 4 and Figure 5 show characteristic pressure traces for two boundary cycles for switching from the 2-stroke to the 4-stroke mode and from the 4-stroke to the 2-stroke mode correspondingly. The instants of the intake and exhaust valve openings and closings are also shown in the figures. In order to find the valve timings which have a significant influence on the IMEP and the combustion phasing during switching we run extensive simulation experiments. By varying the instants of valve closings and openings and comparing the engine outputs recorder on a sufficient interval after the switching we choose the minimal number of inputs for each mode which have a strong influence on the engine outputs. To implement the switching correction from the 4-stroke mode to the 2-stroke mode we choose the intake valve closing (IVC) and the exhaust valve closing (EVC) as our inputs. For the correction after switching to the 4-stroke mode we choose to vary the EVC and the intake valve opening (IVO) simultaneously in opposite direction as they effectively control EGR this way and influence the IMEP and MFB50. For the detailed explanation of influence of valve timings on gas exchange processes in the 2-stroke and the 4-stroke HCCI engine we refer the reader to [5], [13], [14], [15].

With the defined system inputs and outputs we are ready to formulate identification problem. For each of the two modes we would like to find a linear discrete time-invariant model 
of the following form,

$$
\begin{aligned}
x_{m}(k+1) & =A_{m} x_{m}(k)+B_{m} u_{m}(k), \\
y_{m}(k) & =C_{m} x_{m}(k)+D_{m} u_{m}(k),
\end{aligned}
$$

where $k$ stands for the $k$-th engine cycle, and the subscript of the model variables indicate its relation to one of the modes, $m=2-2$-stroke mode, $m=4-4$-stroke mode. For both models the output consists on the IMEP and the combustion phasing and hence $y_{m}(k) \in \mathbb{R}^{2}$ for $m=2,4$. The identification inputs, Figure 3 , for the two modes are different, $u_{2}(k) \in \mathbb{R}^{2}$ (IVC, EVC), and $u_{4}(k) \in \mathbb{R}^{1}$ (EVCIVO). Dimensions of two linear models, $x_{m}(k) \in \mathbb{R}^{n}$, are defined during an identification procedure. Note that the system output, $y_{m}(k)$, at the $k$-th engine cycle depends both on the system state and the inputs applied to the system at the current, $k$-th, cycle.

To identify the two models we apply a subspace identification method to the controlled engine. First, we construct identification signals which are sufficiently exciting and at the same time are representative of control signals used to track reference signals in IMEP and combustion phasing. For that purpose we can use square wave signals with varying period and amplitude. To choose the order of the two linear models we compare the model responses with the responses of the controlled engine. Linear models of order $n=2$ showed a very close matching between the responses for the both modes.

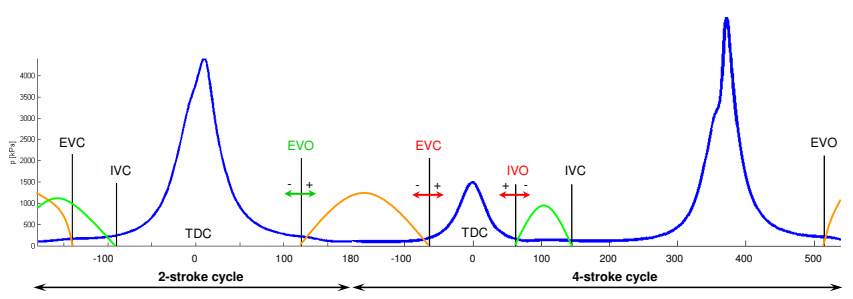

Fig. 4. Characteristic pressure trace for the last 2-stroke cycle and first 4-stroke mode after switching.

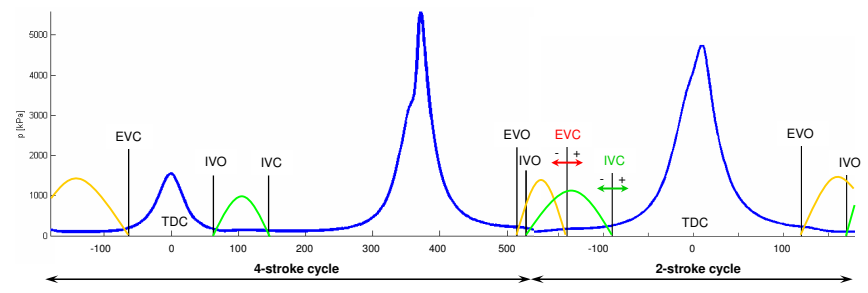

Fig. 5. Characteristic pressure trace for the last 4-stroke cycle and first 2-stroke mode after switching.

With two linear models of the closed-loop system, one for the 2-stroke mode and one for the 4-stroke mode we can express a relationship between the inputs of the engine used to correct for the switching disturbances and the outputs of the engine. In order to simplify the notation we drop the subscripts $m$ defining assignment of variables in the equations (1) to one of two models. For the linear models
(1) we can write,

$$
\begin{aligned}
y(0) & =C x(0)+D u(0), \\
y(1) & =C A x(0)+C B u(0)+D u(1), \\
y(2) & =C A^{2} x(0)+C A B u(0)+C B u(1)+D u(2), \\
\vdots & \\
y(N) & =C A^{N} x(0)+\ldots+D u(N) .
\end{aligned}
$$

If we collect the outputs $y(k)$ and the inputs $u(k)$ and define the collected vectors,

$$
Y_{N}=\left[\begin{array}{c}
y(0) \\
y(1) \\
\vdots \\
y(N)
\end{array}\right], \quad U_{N}=\left[\begin{array}{c}
u(0) \\
u(1) \\
\vdots \\
u(N)
\end{array}\right]
$$

which specify the output and the input of the system on the interval of $N+1$ samples (engine cycles), we can write the equations (2) compactly as,

$$
Y_{N}=A_{x} x(0)+A_{u} U_{N}, \text { where }
$$

$$
A_{x}=\left[\begin{array}{c}
C \\
C A \\
C A^{2} \\
\vdots \\
C A^{N}
\end{array}\right], \text { and }
$$

$$
A_{u}=\left[\begin{array}{ccccc}
D & 0 & 0 & \ldots & 0 \\
C B & D & 0 & \ldots & 0 \\
C A B & C B & D & \ldots & 0 \\
\vdots & \vdots & \vdots & \ddots & \vdots \\
C A^{N-1} B & C A^{N-2} B & C A^{N-3} B & \ldots & D
\end{array}\right] \text {. }
$$

Equation (3) describes a linear mapping between the initial condition of the system, $x(0)$, the input, $U_{N}$, and the output, $Y_{N}$, during the first $N+1$ engine cycles after switching. The initial condition of the linear model, $x(0)$, can describe the disturbance introduced during switching from one mode to another and resulting from the mismatch between the temperatures and the concentrations of species in the engine cylinder. This initial condition depends on the point of switching between the two modes and is equal to zero if the states of the engine working in one mode with a certain output are identical to the states of the engine working in the other mode with the same output.

If we know the desired output of the engine on the horizon of $N+1$ cycles after switching,

$$
Y_{r}=\left[\begin{array}{c}
y_{r}(0) \\
y_{r}(1) \\
\vdots \\
y_{r}(N)
\end{array}\right],
$$

we can formulate an optimization problem of minimizing a quadratic cost function weighting the tracking error and the correction signals. We define the cost function as,

$$
J=\left(Y_{r}-Y_{N}\right)^{\prime} Q_{y}\left(Y_{r}-Y_{N}\right)+U_{N}^{\prime} Q_{u} U_{N} .
$$


In the above expression $Q_{y}$ is a positive-definite matrix weighting the tracking error, $\left(Y_{r}-Y_{N}\right)$, and $Q_{u}$ is a positivedefinite matrix weighting the system inputs, $U_{N}$. The part of the cost function weighting the inputs $U_{N}$ is introduced in order to have a well-defined input. If this part of the cost function is not specified, the correction input can grow unbounded in an attempt to minimize tracking error.

We would like to find the input $U_{N}$ which minimizes $J$. First, we expand the above expression using the equation (3) and then calculate the least squares solution to find the optimal input,

$$
U^{*}=\left(A_{u}^{\prime} Q_{y} A_{u}+Q_{u}\right)^{-1} A_{u}^{\prime} Q_{y}\left(Y_{r}-A_{x} x(0)\right) .
$$

$U^{*}$ minimizes the cost function (4) for a given initial condition $x(0)$ and the reference output $Y_{r}$. Inverse of the matrix $\left(A_{u}^{\prime} Q_{y} A_{u}+Q_{u}\right)$ is well-defined because it is a sum of two matrices, a positive-definite $Q_{u}$ and a positive-semidefinite $A_{u}^{\prime} Q_{y} A_{u}$. In order to find $U^{*}$ we need to know the desired engine output on the interval of $N+1$ cycles after switching and the initial condition, $x(0)$, of the linear engine model which corresponds to a certain switching point for the 2stroke or the 4-stroke mode. In the following we describe how to calculate the initial condition corresponding to a given switching point between the modes. We assume that an engine designer specifies the point of switching between the two modes. If there is more than one point, then we can calculate the initial conditions for all required points and specify look-up table which defines mappings between the desired switching points and the corresponding initial conditions for the linear models. There should be two such tables, one table for each linear system model corresponding to engine controlled by the mode-specific controller in the 2-stroke or the 4-stroke mode.

To find the initial condition, $x(0)$, of the linear model we need to run a new identification experiment. This experiment consists of recording the output of the engine after the hard switching, when no correction eliminating the after-switching oscillations of the output is applied. We need to record the system output on a sufficiently long horizon to allow the system completely react to the disturbance introduced by the switching. If we assume that the output is recorded during $M+1$ engine cycles after switching, then using the linear system model (1) with no inputs we can write,

$$
\begin{gathered}
Y_{M}=A_{M} x(0), \quad \text { where } \\
Y_{M}=\left[y(0)^{\prime} y(1)^{\prime} \ldots y(M)^{\prime}\right]^{\prime},
\end{gathered}
$$

is the collected output vector, and

$$
A_{M}=\left[\begin{array}{c}
C \\
C A \\
C A^{2} \\
\vdots \\
C A^{M}
\end{array}\right] .
$$

If the pair of matrices $A, C$, from the linear system model is observable and the output $Y_{M}$ is recorded on a sufficiently long interval, $M \geq n-1$, ( $n$ in the order of the linear model), then we can find the initial condition which corresponds to the recorded system response. We calculate the initial condition from the least squares solution of equation (6) for $x(0)$

$$
x(0)=\left(A_{M}^{\prime} A_{M}\right)^{-1} A_{M}^{\prime} Y_{M},
$$

Inverse in the above expression is well-defined due to observability of the matrix pair and the sufficiently large $M$. Observability of the pair $A, C$ is guaranteed by the fact that these matrices are the result of the identification procedure described earlier. Hence using equation (7) we can find initial conditions for two linear models and all required switching points.

To be able to calculate the optimal correction sequence $U^{*}$ using equation (5), in addition to the initial condition $x(0)$ we need to know the desired engine output $Y_{r}$ on the interval of $N+1$ engine cycles after switching. If we assume that the mode-specific controllers are tuned to track the desired reference signals in each mode and the optimal correction sequence has to compensate only for the switching disturbance, then $Y_{r}$ can be assigned to zero on the whole $N+1$ interval. This important simplification is a consequence of the separation of tasks between two controller modules represented in Figure 2 and the fact that the two linear models map the switching correction signals into the closed-loop engine outputs. The resulting equation (5) allows calculation of the correction sequences for switching between the two engine modes if the switching points are specified by a designer.

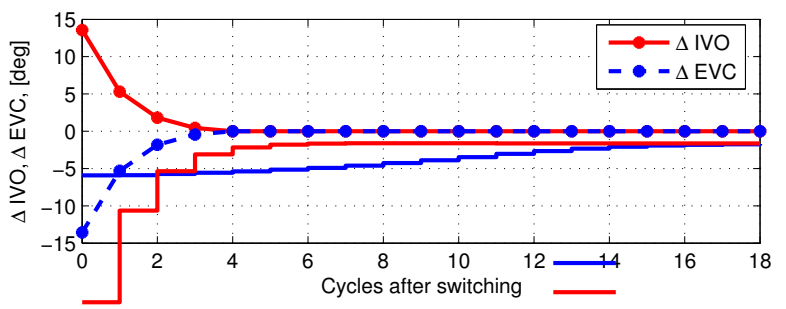

Fig. 6. Switching correction inputs for the 4-stoke mode.

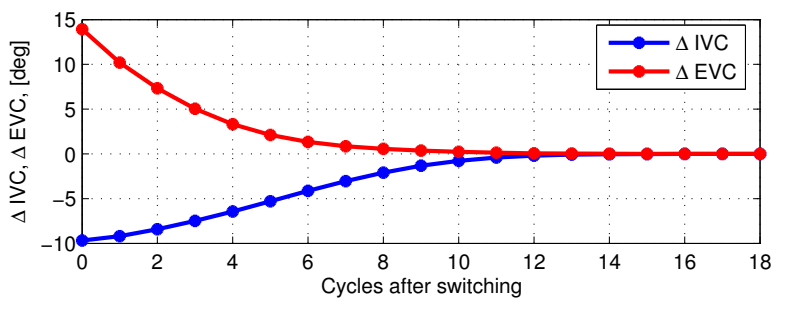

Fig. 7. Switching correction inputs for the 2-stoke mode.

\section{Simulation Results}

We applied the method for calculation of the correction sequences described above to the 19-th order nonlinear model of the HCCI engine. Using equations (5) and (7) we calculated the correction sequences for switching from the 4 -stroke to the 2 -stroke at the point of 4.2 bar IMEP, 
and 3.8 bar IMEP for switching form the 2-stroke to the 4stroke modes. Figures 6 and 7 show the calculated correction sequences. The number of engine cycles after switching for which the correction sequences were applied was specified to be $N=19$. Numerous simulations indicate that the correction sequences represented in the figures are characteristic. The starting points of the correction signals are proportional to the specified switching points and the resulting initial conditions of linear models. Weighting matrices $Q_{u}$ and $Q_{y}$ in the cost function $J$ can be effectively used to tune amplitude and the rate of decay of the correction sequences in order to achieve a desired response of the system to the switching disturbances.

The calculated correction sequences were applied to the nonlinear model of the HCCI engine tracking the ramp input in IMEP and the constant input in MFB50 represented in Figure 1. Figure 8 shows the outputs of the engine during tracking of the reference signals. Both the corrected response and the uncorrected response from Figure 1 are shown. The optimal sequences from Figures 6 and 7 were implemented during switching. As can be seen from the figure the introduced switching correction greatly improves the responses of engine to switching. Oscillations in IMEP and the combustion phasing are reduced significantly. It is important to mention that the responses can be improved further by the appropriate tuning of the correction sequences through the weighting matrices $Q_{y}$ and $Q_{u}$ in the proposed cost function (4). Numerous simulation experiments with various reference signals in IMEP and MFB50 and different switching points showed consistent improvement in tracking the outputs.
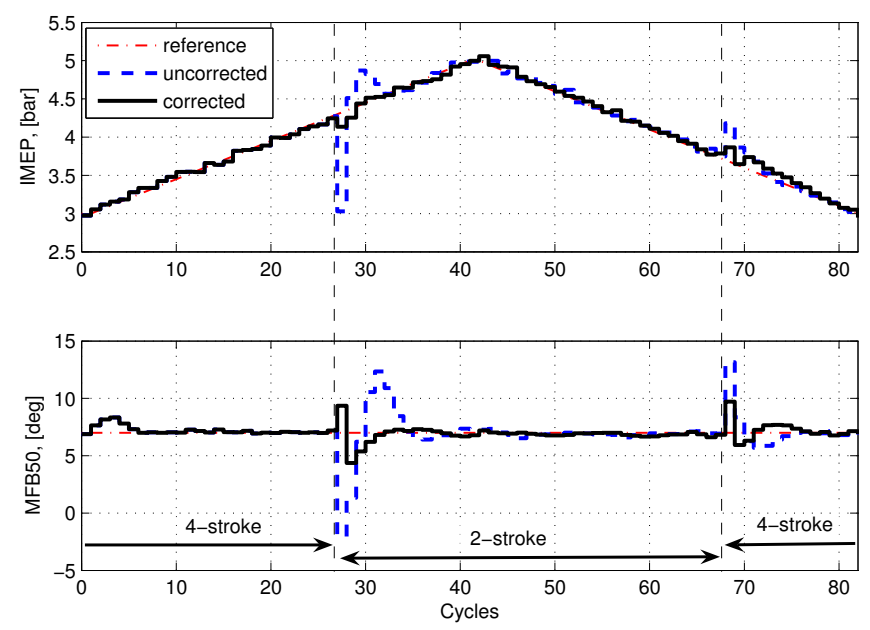

Fig. 8. Uncorrected and corrected outputs of the HCCI engine tracking the ramp reference in IMEP.

\section{CONCLUSION}

In this paper we proposed a structure for a control system which provides smooth transition between the 2-stroke and the 4-stroke modes of HCCI operation. In the proposed structure we used a modular approach for the controller design and separated the tasks of controlling the engine in one of the modes and correcting for the disturbances introduced during switching between two modules: the mode-specific controller and the correcting module. The correcting module generates additional inputs several cycles after the engine switches from one mode to another and compensates for the switching disturbances. We proposed the method for calculation of the correction signals which used basic ideas from the theory of system identification and the theory of optimal control. The method was developed and tested using the 19-th order nonlinear model of the HCCI engine. In the future the proposed control architecture and the method for calculation of the correction sequences have to be validated on an experimental HCCI engine.

\section{REFERENCES}

[1] Caton, P.A., Simon, A.J., Gerdes, J.C., and Edwards, C.F. (2003). Residual-effected homogeneous charge compression ignition at a low compression ratio using exhaust reinduction, Int. J. Engine. Research, $4(3)$.

[2] Chiang, C.J., \& Stefanopoulou, A.G. (2006). Sensitivity analysis of combustion timing and duration of homogeneous charge compression ignition (HCCI) engines, In Proc. of American Control Conference, (pp. 1857-1862).

[3] Fiveland, F.B., \& Assanis, D.N. A quasi-dimensional HCCI model for performance and emission studies. In Proc. of the $9^{\text {th }}$ Int. Conf. on Numerical Combustion, No. MS052.

[4] Karagiorgis, S., Collings, N., Glover, K., \& Petridis, T. (2006) Dynamic modeling of combustion and gas exchange processes for controlled auto-ignition engines. In Proc. of American Control Conference, (pp. 1880-1885).

[5] Knierim, K.L. (2007). Modeling and control of HCCI combustion, Diploma Thesis, Institute of Applied and Experimental Mechanics, University of Stuttgart.

[6] Kojic, A., Hathout, J.P., \& Jasim, A. (2007). Method for extending HCCI load range using a two-stroke cycle and variable valve operation, US Patent 7.231.892.

[7] Koopmans, L., Backlund, O., \& Denbratt, I. (2002). Cycle to cycle variations: their influence on cycle resolved gas temperature and unburned hydrocarbons from a camless gasoline compression ignition engine, SAE 2002-01-0110

[8] Marriott, C., Kong, S.C., \& Reitz, R.D. (2002). Investigation of Hydrocarbon emissions from a direct injection-gasoline premixed charge compression ignited engine, SAE 2002-01-0419.

[9] Martinez-Frias, J., Aceves, S.M., Flowers, D., Smith, J.R., \& Dibble, R. (2000). HCCI engine control by thermal management, SAE 200001-2869.

[10] Milovanovic, N., Blundell, D., Gedge, S., Turner, J. (2005). SI-HCCISI mode transition at diferent engine operating conditions, SAE 2005 01-0156

[11] Haraldson, G., Johanson, B. (2005). Operating conditions using spark assisted HCCI combustion during combustion mode transfer to SI in a multi-cylinder VCR-HCCI engine, SAE 2005-01-0109

[12] Osborne, R.J., Stokes. J., Lake, T.H., Carden, P.J., Mullineux, J.D., Helle-Lorentzen, R., Evans, J.C., Heikal, M.R., Zhy, Y., Zhao, H., Ma, T. (2005). Development of a Two-Stroke/Four-Stroke Switching Gasoline Engine - The 2/4SIGHT Concept, SAE 2005-01-1137

[13] Shaver, G.M., Gerdes, J.C., Jain, P., Caton, P.A., \& Edwards, C.F. (2003). Modeling for control of HCCI engines. In Proc. of American Control Conference, (pp. 749-754).

[14] Shaver, G.M., Roelle, M., \& Gerdes, J.C. (2006). A two-input twooutput control model of HCCI engines. In Proc. of American Control Conference, (pp. 472-477).

[15] Subbotin, M.V., Knierim, K.L., Park, S., Kojic, A., Ahmed, J. (2008). Modeling and control of a two stroke HCCI engine, In Proc. of American Control Conference.

[16] Tunestal, P., Olsson, J.O., \& Johansson, B. (2001). HCCI operation of a multi-cylinder engine, First Biennial Meeting of the ScandinavianNordic section of the Combustion Institute. 